\title{
CONSTRUCCIÓN DE LA TÉCNICA EN PRAXEOLOGÍAS MATEMÁTICAS DADAS EN EL AULA DE TELEBACHILLERATO
}

\author{
David-Alfonso Páez ${ }^{1,2}$, Daniel Eudave-Muñoz ${ }^{2}$, Teresa De Jesús Cañedo-Ortiz ${ }^{2}$, Ana Cecilia \\ Macías-Esparza ${ }^{2}$ \\ 1Consejo Nacional de Ciencia y Tecnología, México; dapaez@correo.uaa.mx \\ ²Universidad Autónoma de Aguascalientes; México; deudave@correo.uaa.mx; tcañedo@correo.uaa.mx; \\ acmacias@correo.uaa.mx
}

\begin{abstract}
Resumen. En México se tiene profesores en Educación Media Superior que, en ocasiones, están obligados a enseñar matemáticas aun cuando su perfil. El presente documento tiene el objetivo de identificar la técnica (procedimientos matemáticos) que enseñar un profesor del Subsistema Telebachillerato para que los estudiantes resuelvan el tipo de tareas relacionado con ecuaciones de primero grado. Esta propuesta es parte un proyecto de investigación, donde participaron trece profesores, de quienes se observó dos clases enseñando contenidos matemáticos de acuerdo con su plan de estudio, esta observaciones tenían la finalidad de hacerlas objeto de discusión con los participantes. En esta investigación mostramos un estudio de casos. Los resultados muestran que el profesor construye una técnica centrada en obtener determinantes para resolver tipo de tareas sobre sistema de ecuaciones, pero la teoría que justifica la funcionalidad se reduce a un discurso más tipo de didáctico, que matemático. En el aula se suelen enseñar técnicas para resolver problema, pero éstas quedan desprotegidas de teorías matemáticas (argumento matemático) que validen su funcionalidad, el alumnos es quien tiene que inferirla o construir su propia teoría a partir de la técnica del profesor.
\end{abstract}

Palabras clave: Práctica Docente; Matemáticas; Álgebra; Educación Media Superior.

\section{CONSTRUCTION OF THE TECHNIQUE OF MATHEMATICAL PRAXELOGIES IN THE TELEBACHILLERATO CLASSROOM}

\begin{abstract}
In Mexico, there are teachers in Higher Middle who, on occasions, are obliged to teach mathematics even when their profile. This document aims to identify the technique (mathematical procedures) to be taught by a teacher in the Telebachillerato Subsystem to solve the type of tasks related to first-grade equations. This proposal is part of a research project, involving thirteen teachers, of whom two classes were observed teaching mathematical content according to their curriculum, these observations were intended to make them the subject of discussion with participants. In this research, we show a case study. The results show that the teacher builds a technique focused on obtaining determinants to solve the type of tasks on the system of equations, but the theory that justifies functionality is reduced to a speech more kind of didactic, than mathematical. Techniques to solve problems are usually taught in the classroom, but these are unprotected from mathematical theories (mathematical argument) that validate their functionality, the student has to infer it or build their own theory from the technique of the teacher.
\end{abstract}

Keywords: Teaching Practice; Mathematics; Algebra; Higher Middle.

\section{INTRODUCCIÓN}

La investigación sobre la práctica docente muestra la importancia de comprender la relación del conocimiento disciplinar y su didáctica, debido a su impacto en el aprendizaje del estudiante (Wilkins, 2008). De acuerdo con Adler, Ball, Krainer, Lin y Novotna (2005) y la 
NCTM (2014), es fundamental que el profesor desarrolle competencias didácticas adecuadas y un conocimiento disciplinar necesario.

En México, la plantilla docente para matemáticas en Educación Media Superior está conformada, en su mayoría, por profesionales de áreas afines a las matemáticas sin una formación docente (Montiel \& Castañeda, 2009). En ocasiones, la formación docente de estos actores educativos es producto de creencias acerca de cómo enseñar y aprender (Guzmán, 2001). Esta situación se agrava en instituciones ubicadas en contexto desfavorable, por ejemplo, el subsistema Telebachillerato, donde su población de estudiantes es de un nivel socio-económico bajo, los docentes suelen tener poca o nula experiencia en la enseñanza de contenidos curriculares (INEE, 2016). Aunado a ello, los profesores de Telebachillerato tienen la responsabilidad de enseñar matemáticas aun cuando su perfil difiere con la asignatura.

El subsistema Telebachillerato surgió ante la escasa atención educativa en comunidades con un alto grado de marginación (Aguilar, Gamboa, \& Farias, 2015). Para los jóvenes que estudian en este subsistema, la escuela y el salón de clases representan espacios ideales para ofrecerles oportunidades que les permitan mejorar su condición; por su parte, para el profesor es un reto enseñar en Telebachillerato, pues tiene que adaptar sus actividades ante las condiciones de sus estudiantes y la propias de la escuela (INEE, 2016). El profesor debe diseñar ambientes de aprendizaje donde los alumnos construyan conocimiento matemático a través de situaciones retadoras; sin embargo, el rezago educativo y los bajos resultados en las últimas evaluaciones nacionales e internaciones, muestran que es necesario tener un aproximación a la práctica docente en este subsistema educativo para entender qué y cómo se enseñan las matemáticas, bajo qué criterios se promueve el aprendizaje en condiciones de pobreza.

En México, la investigación sobre el profesor de Educación Media Superior está relacionada, principalmente, con programas de formación y actualización docente (Martínez-Rizo, 2013). Los estudios que existen acerca de la práctica del profesor de matemáticas de ese nivel educativo están centrados sobre el conocimiento del contenido a enseñar o sobre la resolución de problemas en ambientes de lápiz-y-papel y tecnológico (Sosa, FloresMedrano, \& Carrillo, 2014). En la actualidad, se requieren estudios que den cuenta de la práctica docente en ese nivel, es evidente que el profesor de Educación Media Superior es un factor clave para establecer una conexión entre la Educación Básica y la Superior, pero 
tienen mayores retos aquellos que laborar en instituciones ubicadas en zonas suburbanas, rurales o de pobreza, dadas las condiciones en las que se encuentran (INEE, 2016).

En relación con lo antes expuesto, el presente documento tiene el objetivo de identificar la construcción y validación de técnica de las praxeologías matemáticas que el profesor de Telebachillerato plantea en el salón de clases; en otras palabras, dar cuenta de la técnica matemática que es enseñada y cómo es puesta en práctica. El interés por estudiar al docente de Telebachillerato surge porque es un campo poco abordado y dada su importancia en el proceso de enseñanza y aprendizaje de las matemáticas, el propósito es comprender qué hace y dice en aula para darle sentido a la matemática que construyen en un ambiente cotidiano de clases.

\section{MARCO DE REFERENCIA}

Una teoría que permite tener una aproximación a la práctica y al conocimiento del profesor de matemáticas es la Antropológica de lo Didáctico (Chevallard, 1999; Chevallard, Bosch y Gascón, 1997). Esta propuesta establece que las matemáticas son construidas por un conjunto de sujetos (por ejemplo, el profesor y los alumnos) mediante la práctica y en el seno de una institución. Para Chevallard et al. (1997, p. 51): “Un aspecto esencial de la actividad matemática consiste en construir un modelo (matemático)..., trabajar con dicho modelo e interpretar los resultados obtenidos... para contestar a las cuestiones [resolver tareas] planteadas inicialmente. Gran parte de la actividad matemática puede identificarse, por lo tanto, con una actividad de modelización matemática".

La actividad matemática -y el proceso de estudio de las matemáticas- es parte del conjunto de las actividades humanas y de las instituciones sociales (Chevallard, 1999). Esta actividad puede definirse en términos de Praxeología, donde praxis significa práctica y logos conocimiento. Ambos términos están articulados entre sí: "No hay praxis sin logos, pero tampoco hay logos sin praxis" (Chevallard et al., 1997, p. 274). La praxeología es vista como la práctica en la que surge el conocimiento y está compuesta por cuatro elementos: tipo de tarea, técnica, tecnología y teoría (Figura 1). 


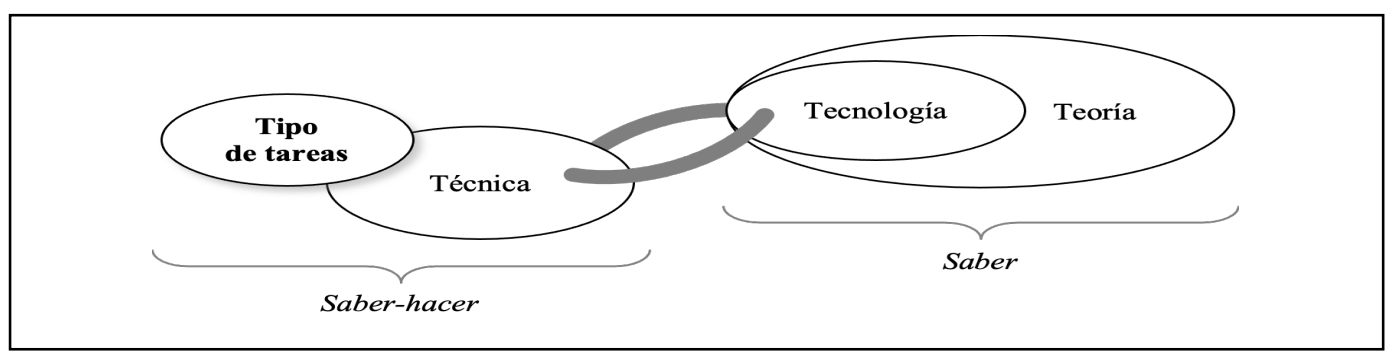

Figura 1. Componentes de una praxeología como actividad matemática construida por una comunidad.

El germen de toda praxeología es la tarea. Chevallard (1999) señala que la tarea se expresa a través de un verbo: calcular, integrar, simplificar, determinar, factorizar, entre otros. Un verbo puede indicar un o varias tareas, pero un Tipo de tarea supone una específico; por ejemplo, determinar los valores de $x$ en la ecuación $x^{2}+3 x=5$ o determinar la o las condiciones en $x$ para que se cumpla la inecuación $2 \leq 3 x<35$. El Tipo de tarea es una construcción institucional que surge de la práctica y es la base de toda praxeología, dado que a partir de ella se dan los otros tres elementos. Para llevar a cabo el tipo de tarea se requiere de, por lo menos, una manera de resolverla, de forma sistematizada y segura (Chevallard et al., 1997). Esta forma recibe el nombre de Técnica y no se refiere necesariamente de tipo algorítmico, pueden ser varias técnicas para un tipo de tarea; sin embargo, en una institución se suele reconocer solo una, excluyendo otras que pueden ser igual de efectivas. La técnica conlleva un discurso racional que justifique su aplicabilidad y validez, definido como Tecnología y tiene tres funciones: a) justificar la técnica para asegurarse de que funciona; b) explicar la técnica con la intención de esclarecerla; c) producir nuevas técnicas. El discurso tecnológico contiene afirmaciones que también debe ser justificado racionalmente, para Chevallard (1999) esta justificación es la Teoría.

Los cuatro elementos que conforman toda praxeología están organizados en dos bloques inseparables: el bloque práctico-técnico que constituye un saber-hacer, y el bloque tecnológico-teórico que se refiere a un saber. La praxeología matemática surge como producto de un proceso de didáctico (estudio de las matemáticas) que es llevado a cabo, en el marco de una institución, por un sujeto o una comunidad de estudio.

\section{METODOLOGÍA}

La presente investigación es de corte exploratorio mediante el estudio de casos (Álvarez, 2003; Schoenfeld, 2008). Por la naturaleza del estudio, participaron trece profesores que 
imparten matemáticas en diferentes escuelas de Telebachillerato, ubicadas en México. La mayoría de los participantes cuenta con estudios de posgrado en educación, en particular, dos de ellos tienen maestría en la enseñanza de las matemáticas; además, la mitad de los participantes tiene al menos ocho años de experiencia docente en este subsistema. Cada profesor, previa autorización, fue observado (videograbación) al enseñar matemáticas a sus estudiantes según el Plan de estudios de Telebachillerato, con la finalidad de que su práctica fuera objeto de discusión al reflexionar sobre ella (Páez, Ramírez, Cañedo, Eudave, Carvajal \& Macías, 2019). Se observaron dos clases por profesor y en diferente momento (Tabla 1). La observación fue no participante para explorar la realidad del salón de clases (Adler \& Adler, 1994), y con ello tener "la oportunidad de recopilar datos 'en directo' de situaciones que ocurren de forma natural” (Cohen, Manion \& Morrison, 2007, p. 396).

Tabla 1. Profesores y contenido trabajado en las clases observadas

\begin{tabular}{lll}
\hline \multirow{2}{*}{ Profesor } & \multicolumn{2}{c}{ Contenido matemático } \\
\cline { 2 - 3 } & \multicolumn{1}{c}{ Primera clase } & \multicolumn{1}{c}{ Segunda clase } \\
\hline Gerardo & Ecuaciones de segundo grado & - \\
Luis & Elipse & Funciones (concepto) \\
Paco & Ecuaciones de primer grado & Teorema de Pitágoras \\
Carlos & Productor notables & - \\
Ana & Factorización & - \\
Julio & Ecuaciones de segundo grado & - \\
Juan & Determinantes para sistema & Teorema de Pitágoras \\
& $2 x 2$ & Ángulos complementarios \\
Rosa & Suma y resta de polinomios & - \\
Cecilia & Características del círculo & Ángulos complementarios \\
Miguel & Productor notables & Ángulos complementarios \\
Pedro & Productos notables & Ángulos complementarios \\
Carla & - &
\end{tabular}

Fuente: Páez et al. (2019).

En un primer momento se videograbaron once clases, en la segunda observación fueron siete. Algunos de los profesores que participaron en la primera observación decidieron no hacerlo en la segunda. Las observaciones de aula se llevaron a cabo entre noviembre de 2017 y febrero de 2018, para ello se determinó con los profesores la fecha de videograbación, se respetó el plan de trabajo (contenido a enseñar y el tiempo destinado). Aunque se utilizaron videocámaras para esta actividad, se buscó que las observaciones no afectaran el escenario y el contexto natural del proceso de enseñanza y aprendizaje que se estaba dando en ese momento.

El análisis de las clases estuvo centrado en identificar los cuatros componentes de las praxeologías construidas en las dos clases observadas por los profesores, y cómo es la relación entre estos elementos. Para el presente documento, se presentan un caso de 
estudio: el profesor Julio dando Ecuaciones de primer grado a estudiantes de primer semestre de Telebachillerato, quien muestra la construcción y validez de la técnica para resolver ecuaciones lineales.

\section{ANÁLISIS DE RESULTADOS}

El libro de texto es un recurso fundamental para el profesor de Telebachillerato, ya que le proporciona los tres primeros elementos de la praxeologia: tipos de tarea, tecnicas y tecnología para el tema de ecuaciones de primero grado (SEP, 2015). El libro de texto propone cuatro técnicas para resolver los tipos de tareas especificos en torno a este contenido matemático: método de igualación, determinantes, reducción y gráfico (Cfr. SEP, 2015, pp. 282-310). En las clases observadas, las praxologías construidas por Julio estan centradas en trabajar los métodos de determinación y reducción. Para los tipos de tareas $a x+b y+c=0$, Julio muestra la técnica que los estudiantes deben utilizar, y las cual está centrada en usar los coeficientes de las ecuaciones (Figura 2).

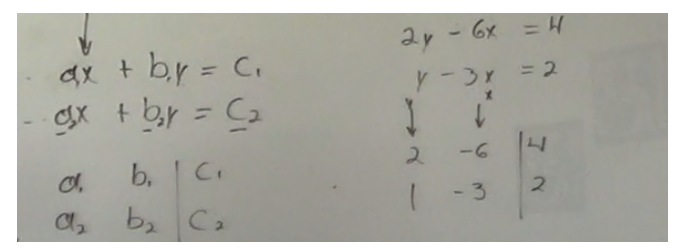

Figura 2. Tipo de tarea: Calcular los valores de las incógnitas en el sistema de ecuación de primer grado.

La técnica está centrada en obtener tres determinantes, los cuales Julio los define como Dp (Determinante principal), Da (Determinante del coeficiente a) y Db (Determinante coeficiente b). Para obtener cada determinado, Julio plantea una relación cruzada entre los coeficientes de la primera ecuación con los de la segunda. La relación involucra multiplicaciones y restas de los resultados. La construcción de la técnica a través de determinantes lleva a identificar las relaciones entre parejas cruzadas de los coeficientes para obtener un tercero:

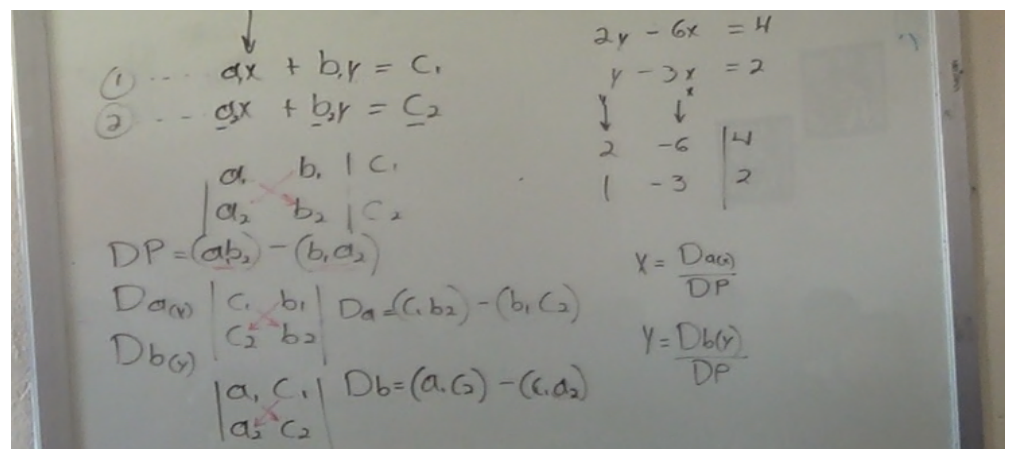

Figura 3. Técnica: relación cruzada entre parejas los coeficientes de las ecuaciones 
En esta técnica, Julio destaca que Da y Db permitirán obtener los valores de las incógnitas $\mathrm{x}$ e y al dividir el resultado de la relación con el valor de Dp. Es evidente que el discurso que Julio está centrado en el procedimiento de solución es una teoría que sustente la funcionalidad de esta técnica. Julio explica paso a pasa cómo desarrollar la técnica, sin dar cuenta de por qué de ella. La Técnica involucra obtener primero DP, para después obtener los otros dos determinantes. En la construcción que desarrolla Julio en técnica, a su vez, se muestra la teoría que le da sentido. La teoría que propone Julio se relaciona con alinear los coeficientes de las ecuaciones de acuerdo con las laterales, el cual se refiere a columnas y filas tomando a, b, c. Julio afirma que es fundamental tal alineamiento de los coeficientes para poder usar la técnica, él les dice a los estudiantes: "Si se fijan se generan columnas y se generan filas. Esta columna [la primera] se refiere a la letra $x$, esta columna [la segunda] es la letra y, y la otra columna es la letra c. Una segunda teoría que también da Julio es el acomido de los datos para obtener los valores de los determinantes, Julio plantea que "es importante tomar de izquierda a derecha" y de "derecha a izquierda" los datos de las ecuaciones como se muestra la Figura 3. Al parecer en la justificación se deja de lado el principio de conmutación, el cual plantea que el orden no altera el producto; es decir, $a_{1} b_{2}=$ $b_{2} a_{1}$.

\section{CONCLUSIONES}

Los resultados muestran que Julio tiene interés de que los estudiantes comprendan cómo resolver ecuaciones lineales de primero grado; para ello, él propone la técnica de determinantes según el plan de estudio. La técnica se apega a lo institucional es un procedimiento que involucra dos pasos: obtener los determinantes y después generar divisiones. La teoría que Julio es más de tipo didáctico, en términos de que los estudiantes hagan uso correcto de la técnica: acomodar los datos de tal forma que la técnica funcione. La construcción de la teoría se aleja del discurso matemático, tal como probar y argumentar de manera matemática la técnica, en este sentido se dejan huecos de por qué se requiere obtener un determinante principal y cuál es la funcionalidad de la técnica. Los estudiantes deben construir o inferir la teoría matemática que está implícita en la técnica de Julio, por ejemplo, que el determinante principal es necesario y juega papel para obtener los valores $\mathrm{x}$ e y al dividir otros determinantes entre éste. Tal vez, la falta de teoría matemática se debe a que Julio se apegan al plan de estudios, donde el objetivo es enseñar técnicas (SEP, 2015). 
Agradecimientos. Esta investigación se desarrolló dentro del Programa de Investigaciones Educativas de la Universidad Autónoma de Aguascalientes, México, PIE 17-7. Agradecemos la colaboración de la Coordinación de Telebachilleratos en Aguascalientes, de los profesores que participan en el proyecto, y de las asistentes Martha Cinthia García Gaytán y María Guadalupe Capetillo Plascencia.

\section{REFERENCIAS}

Adler, P. A. \& Adler, P. (1994). Observational techniques. En N. K. Denzin \& Y. S. Lincoln (Eds.), Handbook of qualitative research (pp. 377-92). Londres: Sage Publications.

Adler, J., Ball, D. L., Krainer, K., Lin, F. L. \& Novtona, J. (2005). Reflections on an emerging field: researching mathematics teacher education. Education Studies in Mathematics, 60, 359-381.

Álvarez, J. L. (2003). Cómo hacer investigación cualitativa. Fundamentos y metodología. México: Paidós Educador

Chevallard, Y. (1999). L'analyse des pratiques enseignantes en théorie anthropologique du didactique. Recherches en Didactique des Mathématiques. 19, 221-266.

Chevallard, Y., Bosch, M. \& Gascón, J. (1997). Estudiar matemáticas: el eslabón perdido entre enseñanza y aprendizaje. Horsori/ICE.

Cohen, L., Manion, L. \& Morrison, K. (2007). Research methods in education. New York: Routledge.

Guzmán, M. L. (2001). Formación, concepciones y práctica de los profesores de matemáticas. Educación Matemática, 13(3), 93-106.

INEE (2016). La educación obligatoria en México. Informe 2016. México, DF: INEE. Disponible en http://publicaciones.inee.edu.mx/buscadorPub/P1/I/241/P1/241.pdf

Martínez-Rizo, F. (2013). Dificultades para implementar la evaluación formativa: revisión de literatura. Perfiles Educativos, 35(139), 128-150.

Montiel, G., \& Castañeda, A. (2009) Educación a distancia en-línea: elementos para la formación del docente de matemáticas en servicio. Innovación Tecnológica UPVM, 1, 20-28.

NCTM (2014). Principles to Actions: Ensuring Mathematical Success for All. USA: NCTM.

Páez, D. A., Ramírez, M. M. E., Cañedo, T. J., Eudave, D., Carvajal, M. \& Macías, A. C. (2019). Enseñar matemáticas en contexto vulnerable: retos y dificultades del profesor de Telebachillerato. En Atas Investigação Qualitativa em Educação/Investigación Cualitativa en Educación. Desponibble en https://proceedings.ciaiq.org/index.php/CIAIQ2019/article/view/2294

Schoenfeld, A. (2008). Research methods in (mathematics) education. En L. D. English (Ed.), Handbook of international research in mathematics education (pp. 467-519). USA: Routledge Taylor \& Francis Group.

SEP (2015). Matemáticas I. Telebachillerato. México: SEP. Desponible en www.dgb.sep.gob.mx/ servicios-educativos/telebachillerato/LIBROS/1-semestre-2019/Matematicas-I.pdf

Sosa, L., Flores-Medrano E. \& Carrillo J. (2014). Conocimiento del profesor acerca de las características de aprendizaje del álgebra. Enseñanza de las Ciencias, 33.2, 173-189.

Wilkins, J. L. M. (2008). The relationship among elementary teachers' content knowledge, attitudes, beliefs, and practices. Journal in Mathematics Teacher Education, 11, 139-164. 